\title{
Investigation of the Arrhythmia Parameters of Hemodialysis Patients with Hypotensive Episodes
}

\author{
Zafer Yalım ${ }^{* 1}$, Mehmet Emin Demir ${ }^{2}$, Çağlar Alp ${ }^{3}$, Melike Özkan ${ }^{4}$, Sümeyra Alan Yalım ${ }^{5}$ \\ ${ }^{1}$ Afyonkarahisar Healty Science University, Department of Cardiology, Afyonkarahisar/Türkiye \\ ${ }^{2}$ İstinye University, Department of İnternal Medicine, İstanbul \\ ${ }^{3}$ Kırıkkale University, Department of Cardiology, Kırıkkale \\ ${ }^{4}$ Kırıkkale University, Department of İnternal Medicine, Kırıkkale \\ ${ }^{5}$ Afyonkarahisar State Hospital, Department of İnternal Medicine, Afyonkarahisar \\ Email id - ${ }^{1}$ zaferyalm@yahoo.com.tr, ${ }^{2}$ demirmehmetemin@hotmail.com, ${ }^{3}$ drcaglaralp@gmail.com, \\ ${ }^{4}$ melikeozkan89@yahoo.com, ${ }^{5}$ alansumeyra@gmail.com
}

*Corresponding Author -Zafer Yalim, M.D.

Received 01 December 2018;

Accepted 25 January 2019;

Published 01 February 2019

\begin{abstract}
Background: Sudden cardiac death is the leading cause of cardiac caused death in hemodialysis patients. Hypotensive episodes in pre- intra and post-dialytic periods are the serious clinical challenges that effect patients life quality and prognosis. We investigated whether hypotensive episodes have an association with ventricular arrhythmias and discuss literature. Methods: Totally 56 cases; twenty-two patients with frequent hypotension episodes, and 15 normotensives patients who were on maintenance hemodialysis treatment and 19 healthy individuals were included in the study. Pre-intra and post-dialytic periods recorded by using a 24 -h Holter rhytm device. $p<0,05$ is postulated as significantly distinct. Results: Records of 50 participants were evalated properly. Both in hypotensive and normotensive hemodialysis patients ventricular extrasystoles [VES] counts were in a strong relation with lower blood pressure $\mathrm{p}<0,05$. Comorbid diseases [diabetes mellitus, coronar artery disease] which may lead arrhtymias were similar in all three groups. 16 of 18 hyptensive patients also were non-dipper [p<0,05]. Conclusion: Our study revealed that frequent hypotensive episodes are associated with arrhythmia. Clinicians should be aware of arhhythmia which is most important cause of sudden cardiac death and must consider performing a deep cardiological evaluation.
\end{abstract}

Keywords: Arrhythmia, Hemodialysis, Hypotension

\section{Introduction}

Hemodialysis is the most common treatment option of renal replacement treatment in patients with end-stage kidney disease. Sudden cardiac death is the main short- and long-term cause of death in hemodialysis population and also it is being assumed that arrhythmia is the main promotor factor in the sudden cardiac death of the hemodialysis patients ${ }^{[1,2]}$. Hemodialysis patients, due to their conventional milieu, have many risk factor for cardiac death. It is mandatory to determine each factor which can lead to cardiac caused death in hemodialysis patients, and, in the light of the wellproven data, preventing of, or at least trying to treat of arrhythmia might prolong the surviving of HD patients.

A group of patients is suffering from hypotensive episodes in preintra or post-dialytic period. Intra-dialytic hypotensive episodes commonly have been linked to sudden volume changes. Besides, rapid electrolyte changes, heart failure, and autonomic dysfunction may contribute to hypotensive episodes ${ }^{[3]}$. The hypotensive course persssists after hemodialysis sessions in many patients. Even, those patients have a tendency to be hypotensive or at the lower limit of normal range of blood pressure in pre-dialysis periods.

Hypotensive episodes cause to a vicious circle combined of "hypotension-hemodialysis inadequacy-hypervolemia". Each of these factors strongly impacts mortality in hemodialysis patients. But the promoting factor for hypotension is not clear.

We aimed to investigate whether the recurrent hypotension periods [which proven by office, home or 24-h ABPM measurements] in hemodialysis patients is associted with arrhytmia and discuss the literature in the light of our data. 


\section{Methods}

Totally 56 cases; twenty-two patients with frequent hypotension episodes (proven by office-home and/or 24-hour ambulatory blood pressure monitoring), and 15 normotensives patients who were on maintenance hemodialysis treatment and 19 healthy individuals were enrolled in the study. Patients have received hemodialysis treatment at afternoon sessions. At the same day, at 08:00 pm, all participants were invited to install a 24-hour Holter rhythm followup device. Thus, pre-intra and post-dialytic periods were included to obtain likely arrhythmias. Four patients in the hypotension group and two in the control group were excluded due to insufficient records of 24-h Holter rhythm follow-ups. Local ethics committee approval and informed consent form were obtained from all participants. S.P.S.S. version 23.0 statistical package program were used to analyze data. $\mathrm{P}<0,05$ is postulated as significantly distinct.

\subsection{Exclusion Criteria;}

- $\quad$ Receiving anti-hypertensive drug
- Receiving anti-arrhythmic drug

- Clinically hypovolemic patients (suspected patients reevaluated with bioimpedance study)

- Hemodialysis patients who receives treatment twiceweekly

- Abnormal endocrin panel (abnormal level of plasma renin activity, cortisol, thyroid stimulating hormone, aldosterone)

- $\quad$ Patients with abnormal ECG findings occured in recent 3 months.

\section{Results}

Patients and controllers' demographic and laboratory results were given in table-1. Patients who have frequent hypotensive episodes were detected as normotensive in 24-h ABPM studies. Besides, this group had including substantially non-dipper (table-2) and arrhytmic patients, compared to normotensives patients and controls.

Table-1: Demographic and laboratory parameters of participantas

\begin{tabular}{|c|c|c|c|c|}
\hline & $\begin{array}{c}\text { Hypotensive-HD } \\
n=18\end{array}$ & $\begin{array}{c}\text { Normotensive-HD } \\
n=15\end{array}$ & $\begin{array}{c}\text { Control } \\
\mathbf{n}=17\end{array}$ & $P$, value \\
\hline Age, year & $57 \pm 8,90$ & $58,63 \pm 10,77$ & $54,84 \pm 18,34$ & $=0,785$ \\
\hline Sex, male/female & $9 / 9$ & $10 / 5$ & $5 / 12$ & $>0,05$ \\
\hline Coronary artery disease, exist/absent & $5 / 12$ & $10 / 8$ & $6 / 11$ & $>0,05$ \\
\hline Diabetes mellitus, exist/absent & $7 / 8$ & $8 / 10$ & $5 / 12$ & $>0,05$ \\
\hline Creatinin, $\mathbf{m g} / \mathbf{d l}^{*}$ & $7,34 \pm 3,49$ & $8,35 \pm 1,71$ & $0,77 \pm 0,21$ & $<0,001$ \\
\hline Potassium, mEq/lt & $5,05 \pm 1,10$ & $5,27 \pm 0,66$ & $4,67 \pm 0,39$ & $>0,05$ \\
\hline Sodium, mEq/lt*** & $137,60 \pm 2,79$ & $137,54 \pm 2,76$ & $139,14 \pm 2,44$ & $<0,05$ \\
\hline Calcium, mEq/lt & $8,70 \pm 0,57$ & $8,82 \pm 0,98$ & $8,92 \pm 0,71$ & $>0,05$ \\
\hline $\begin{array}{l}\text { Left ventricular hypertrophy } \\
\text { Yes/no }\end{array}$ & $10 / 8$ & $9 / 6$ & $9 / 8$ & $>0,05$ \\
\hline Pre-dialysis systolyc BP & $87,80 \pm 7$ & $126,05 \pm 17,12$ & & $<0,001$ \\
\hline Pre-dialysis diastolic BP & $61,2 \pm 5,57$ & $80,26 \pm 11,72$ & & $<0,001$ \\
\hline Post-dialysis systolic BP & $84,60 \pm 9,85$ & $109,63 \pm 15,76$ & & $<0,001$ \\
\hline Post-dialysis diastolic BP & $55,70 \pm 7,61$ & $67,72 \pm 10,37$ & & $<0,001$ \\
\hline Average heart rate & $77,9 \pm 6,93$ & $80,09 \pm 11,54$ & $72,89 \pm 8,91$ & $=0,111$ \\
\hline Number of VES & $907,60 \pm 682,57$ & $198,45 \pm 146,41$ & $69,05(0-418)$ & $<0,001$ \\
\hline $\begin{array}{l}\text { ABPM-daily, systolyc BP } \\
\text { diastolic BP }\end{array}$ & $\begin{array}{l}101,88 \pm 53 \\
64,18 \pm 9,06\end{array}$ & $\begin{array}{l}119 \pm 15,47 \\
78,63 \pm 9,88\end{array}$ & $\begin{array}{c}128,31 \pm 31,58 \\
82,68 \pm 14,14\end{array}$ & $\begin{array}{l}<0,001 \\
<0,001\end{array}$ \\
\hline $\begin{array}{l}\text { ABPM-night, systolyc BP } \\
\text { diastolic BP }\end{array}$ & $\begin{array}{c}99,47 \pm 14,50 \\
65,34 \pm 8,74\end{array}$ & $\begin{array}{c}101,27 \pm 13,63 \\
67,27 \pm 7,32\end{array}$ & $\begin{array}{c}108,84 \pm 17,42 \\
71,68 \pm 12,74\end{array}$ & $\begin{array}{l}=0,246 \\
=0,272\end{array}$ \\
\hline $\begin{array}{l}\text { ABPM-24-h, systolyc BP } \\
\text { diastolic BP }\end{array}$ & $\begin{array}{c}100,03 \pm 13,42 \\
64,80 \pm 8,62\end{array}$ & $\begin{array}{c}110,09 \pm 13,42 \\
73,36 \pm 12\end{array}$ & $\begin{array}{c}118,26 \pm 16,79 \\
77,84 \pm 13,30\end{array}$ & $\begin{array}{l}<0,05 \\
<0,05\end{array}$ \\
\hline
\end{tabular}

HD; hemodialysis, BP; blood pressure, VES; ventriculary extra-systol ABPM; ambulatory blood pressure monitoring, *Distinctions exist between all groups, **Distinctions exist only between hyptensive-HD versus Control and Normotensive-HD versus Control

Table 2: Comparison of non-dipper individuals among all groups

\begin{tabular}{|c|c|c|c|c|c|}
\hline & & \multicolumn{2}{|c|}{ Non dipper } & \multirow{2}{*}{ Total } & \multirow{2}{*}{$P$, value } \\
\hline & & Yes & no & & \\
\hline \multirow{3}{*}{ CASE } & Hypotensives-HD $^{1}$ & 16 & 2 & 18 & 1 vs $2=p<0,05$ \\
\hline & Controls $^{2}$ & 5 & 12 & 17 & 2 vs $3=p<0,05$ \\
\hline & Normotensives-HD $^{3}$ & 7 & 8 & 11 & 1 vs $3=p<0,05$ \\
\hline \multicolumn{2}{|l|}{ Total } & 28 & 22 & 50 & \\
\hline
\end{tabular}

Average ejection fraction in hypotensive group was $57 \%$ (in a range of 40-65\%). All patients, except one patient in hypotensive group, had a preserved systolic function.

Both in hypotensive and normotensive hemodialysis patients VES counts were in a strong relation with lower blood pressure.
Blood pressure changes during dialysis (pre-and post-dialysis systolic and diastolic blood pressure) were significant; $p<0,001$, both in hypotensives and normotensives HD patients.

We didn't not life-threatening ventricular arrhythmia, atrial fibrillation or supraventriküler arrhythmia in the ECG Holter 
recordings. Monitored VES were frequently at a characteristic of monomorphic.

\section{Discussion}

Sudden cardiac death is the major cardiovascular caused death in hemodialysis population and arrhythmia is the leading cause of sudden cardiac death. Hemodialysis patients are vulnerable to arrhythmia due to various comorbid diseases. Our trial demonstrated that frequent hypotensive episodes are strongly associated with ventricular arrhythmias and may address to an early stage of sudden cardiac death.

SCD is the unexpected natural death from a cardiac cause within one hour of onset of symptoms in a person not known previously to have a potentially fatal condition ${ }^{[4]}$ and accounts for $6-13 \%$ of all deaths, in the general population ${ }^{[5]}$. In contrast, SCDs are more prevalant in hemodialysis population and account for $\neg \sim \% 25$ of all-cause deaths than general population ${ }^{[6]}$. A striking point to care that is defined in SHARP study (Study of Heart and Renal Protection) is overall survival with statin treatment did not improve in patients with chronic kidney disease ${ }^{[7]}$. So it is reasonable to think the death from the cardiac origin is primarily due to arrhythmias in hemodialysis patients.

Compare to general population, a burden diseases have been accumulated on hemodialysis patients. Coronary artery disease, left ventricular failure and hypertrophy, hyperkalemia, diabetes mellitus and cardiac autonomic dysfunction, vascular calcification and dialysis itself carries on high risk of cardiac caused death. We compared relatively homogenous groups who had a similar disease burden (diabetes mellitus, coronary artery disease, LVH), age, gender, and serum electrolytes (potassium and calcium) and have found hypotensive episodes is strongly associated with ventricular extrasystoles.

In the past, ventricular extrasystoles have been examined in hemodialysis patients, by using various study design and outcomes. Kitano et al. had selected hemodialysis patients who hadischemic changes on exercise stress test and thereafter divided the patients into two groups as stenotic and non-stenotic after performing coronary angiography. The stenotic group had experienced more frequent ventricular arrhythmias than non-stenotic both during hemodialysis and for $12 \mathrm{~h}$ after dialysis ${ }^{[8]}$. Abe et al. had enrolled the hemodialysis patients who had a history of arrhythmia and $\mathrm{CAD}$ in their study and reported that ventricular premature beats are a consequence of the rapid potassium change during hemodialysis sessions ${ }^{[9]}$. Canziani et al had compared hemodialysis and peritoneal dialysis patients and found HD patients have more arrhythmias. But LVH was a confounding factor in their study ${ }^{[10]}$. Radaelli et al. have found no difference in outcome after 4-year follow-up of HD patients with arrhythmia ${ }^{[11]}$. In today ventricular extrasystoles are risk factors for de novo heart failure, and this issue have to been questioned entirely once again.

Hypotension during hemodialysis affects $5-10 \%$ of hemodialysis patients and is more prevalent among patients on long-term hemodialysis. Although its pathophysiology is not clear, chronic hypotension (a systolic blood pressure $<100 \mathrm{mmHg}$ ) is assumed to occurs due to reduced response to vasopressor agents ${ }^{[12]}$ and accumulation of vasodilator nitrites/nitrates ${ }^{[13]}$. Interestingly, those with hypotension had a preserved systolic function [normal ejection fraction], as it is in our study.
The association between hypotension and arrhythmia in hemodialysis patients has not been investigated broadly. Literature has been focused on chronic intradialytic hypotension. The evaluation of hypotension episodes which occurs in dialysis-free days or the evaluation of the lower limit of normal range of blood pressure in the predialysis period has been neglected.Friedrich et al. reported that predialysis hypotension [defined as systolic blood pressure $<110 \mathrm{mmHg}$ ] increaes 1.86 fold mortality, in contrast, predialysis hyertension have no impact on all cause mortality and they have pointed out to the importance of the care of those group of hypotensive patients ${ }^{[14]}$. Our study cohort were selected from patients who had hypotension episodes both in dialysis-free periods and in intradialytic periods and records were obtaied from home and 24-h ABPM measurements. We demonstrated that being hypotensive or being at lower limit of normal blood pressure range is associated with ventricular arrhythmias, despite the preserved cardiac systolic function.

One study demonstrated increased heart rate variability [HRV] due to sympathic activitiy in hypotensive episodes during hemodialysis sessions $\left[{ }^{15]}\right.$. We did not do observe HRV in an intra-dialytic and dialysis-free period in our patients.

The available observational studies suggest that in hemodialysis patients the relation between BP and outcomes is complex and differs from the general population. First challenge is that it remains unclear which blood pressure to use in mortality studies; pre, intra or pos-dialytic levels?. Second; the blood pressure may be substantially distinct from the first day of the hemodialysis session to the last session of the week, also may it differs in dialysis and dialysis-free days ${ }^{[16]}$. Patients whose systolic blood pressure fell with hemodialysis had a significantly decreased risk of hospitalization or death at 6 month ${ }^{[17]}$. But the outcome in hypotensive HD patients may be considerably different and, interestingly, reduction amount of blood pressure, both systolic and diastolic, in patients with hypotensive episodes was lower than normotensive HD patients. It can explain why those patients more tolerant to hemodialysis sessions.

A well-known issue is that intradialytic hypotension leads to impairment of the myocardial perfusion and is associated with a worse prognosis ${ }^{[17,18]}$. Park et all. reported that modest declines in BP after hemodialysis are associated with the greatest survival, whereas any rise or large decline in BP is associated with worsened survival $^{[18]}$. So, it may be difficult to adjust the hemodialysis prescription of an HD patient who has low level of blood pressure in predialysis period. Those patients may recieve inadequate hemodialysis treatment and may remain in a hypervolemic state which is the other mortality factor. Our patients were evaluated with a bioimpedance analyze device and have been found euvolemic. They well tolerated hypotensive episodes and did not develop symptoms, but accompanying arrhythmias are also a big concern and may be deserve further cardiologic investigations.

Being non-dipper is a mortality factor both in general CKD patients and hemodialysis population ${ }^{[19-21]}$. Almost all HD patients with hypotensive episodes were non-dippers in our study. Almost half of the control group were consisted of non-dippers, as including of patients with diabetes mellitus and coronary artery disease, although, number of non-dippers considerably were higher in hypotensive and normotensive HD groups. 
Finally, hypotensive episodes, both in intradialytic and extradialytic periods may be a sign of serious ventricular arrhythmias and worse prognosis. Those patients may tolerate hemodialysis session with a large amount of ultrafiltration volume, however, a deep cardiological evaluation is mandatory for keeping them with a longer surviving.

\section{References}

[1] J. Tong et al., "Mortality and Associated Risk Factors in Dialysis Patients with Cardiovascular Disease.," Kidney Blood Press. Res., vol. 41, no. 4, pp. 479-487, 2016.

[2] D. Green et al., "Sudden cardiac death in hemodialysis patients: an in-depth review," Am. J. Kidney Dis., vol. 57, no. 6, pp. 921-929, 2011.

[3] D. Berger and J. Takala, "Hypotension and hypovolemia during hemodialysis: is the usual suspect innocent?," Crit. care, vol. 20, no. 1, p. 140, 2016.

[4] F. Members et al., "2015 ESC Guidelines for the management of patients with ventricular arrhythmias and the prevention of sudden cardiac death: The Task Force for the Management of Patients with Ventricular Arrhythmias and the Prevention of Sudden Cardiac Death of the Europe," Eur Hear. J. 2015 Nov 1;36(41), p. $2793-2867$.

[5] S. S. Chugh et al., "Current burden of sudden cardiac death: multiple source surveillance versus retrospective death certificate-based review in a large US community," J. Am. Coll. Cardiol., vol. 44, no. 6, pp. 1268-1275, 2004.

[6] Rich, "Morbidity and mortality. in: USRDS 2008 Annual Data Report. National Institutes of Health, National Institute of Diabetes and Digestive and Kidney Diseases, Bethesda, MD; 2008: 129-146."

[7] "The Sharp Collaborative Group. Should we reduce LDL cholesterol in patients with chrnic kidney disease? (The reults of the Study of Heart and Renal Protection (SHARP). ASN Renal Week, Denver, CO); November 20, 2010."

[8] Y. Kitano et al., "Severe coronary stenosis is an important factor for induction and lengthy persistence of ventricular arrhythmias during and after hemodialysis," Am. J. kidney Dis., vol. 44, no. 2, pp. 328-336, 2004.

[9] S. Abe et al., "Electrocardiographic abnormalities in patients receiving hemodialysis," Am. Heart J., vol. 131, no. 6, pp. 1137-1144, 1996.
[10] M. E. Canziani et al., "Hemodialysis versus continuous ambulatory peritoneal dialysis: effects on the heart," Artif. Organs, vol. 19, no. 3, pp. 241-244, 1995.

[11] B. Radaelli, A. Cavalli, and R. Latini, "Multicentre, cross-sectional study of ventricular arrhythmias in chronically haemodialysed patients," Lancet, vol. 332, no. 8606, pp. 305-309, 1988.

[12] Cases and E. Coll, "Chronic hypotension in the dialysis patient.," J. Nephrol., vol. 15, no. 4, pp. 331-335, 2002.

[13] E. Coll, M. Larrousse, S. Collado, W. Jiménez, and A. Cases, "Chronic hypotension in hemodialysis patients: role of functional vascular changes and vasodilator agents.," Clin. Nephrol., vol. 69, no. 2, pp. 114-120, 2008.

[14] F. K. Port et al., "Predialysis blood pressure and mortality risk in a national sample of maintenance hemodialysis patients," Am. J. kidney Dis., vol. 33, no. 3, pp. 507-517, 1999.

[15] H. Fukuta et al., "Prognostic value of heart rate variability in patients with end-stage renal disease on chronic haemodialysis," Nephrol. Dial. Transplant., vol. 18, no. 2, pp. 318-325, 2003.

[16] J. K. Inrig et al., "Association of intradialytic blood pressure changes with hospitalization and mortality rates in prevalent ESRD patients," Kidney Int., vol. 71, no. 5, pp. 454-461, 2007.

[17] B. V Stefánsson et al., "Intradialytic hypotension and risk of cardiovascular disease," Clin. J. Am. Soc. Nephrol., vol. 9, no. 12, pp. 2124-2132, 2014.

[18] J. Park et al., "A comparative effectiveness research study of the change in blood pressure during hemodialysis treatment and survival," Kidney Int., vol. 84, no. 4, pp. 795-802, 2013.

[19] X. Che et al., "The impact of non-dipper circadian rhythm of blood pressure on left ventricular hypertrophy in patients with non-dialysis chronic kidney disease," Acta Cardiol., vol. 72, no. 2, pp. 149-155, 2017.

[20] Chaudhuri et al., "Role of twenty-four-hour ambulatory blood pressure monitoring in children on dialysis," Clin. J. Am. Soc. Nephrol., p. CJN-07960910, 2011.

[21] M. Rahman, V. Griffin, R. Heyka, and B. Hoit, "Diurnal variation of blood pressure; reproducibility and association with left ventricular hypertrophy in hemodialysis patients," Blood Press. Monit., vol. 10, no. 1, pp. 25-32, 2005. 